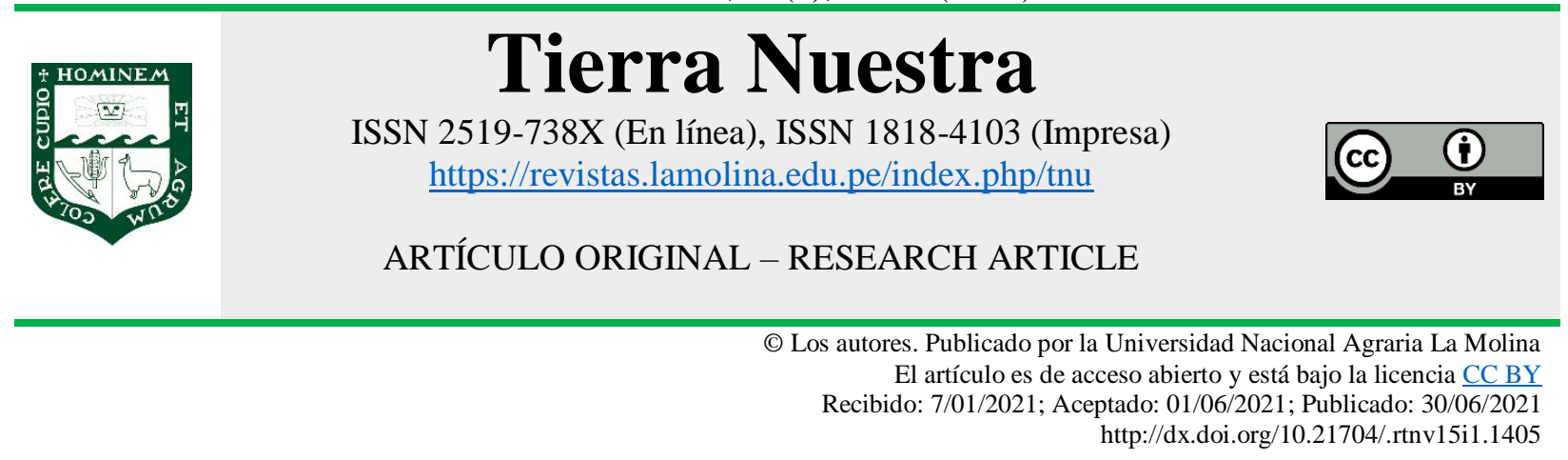

\title{
Redes radiales y prototipicidad en los conceptos cromáticos aimaras
}

\section{Redes radiales y prototipicidad en los conceptos cromáticos aimaras}

\author{
Jaime Edilberto Huanca Quispe ${ }^{1 *(1)}$ \\ * Autor de correspondencia: jhuancaq@unmsm.edu.pe
}

\section{RESUMEN}

En la lengua aimara se registran vocablos relativos a la cromaticidad, muchos de estos nombres están asociados con diferentes escalas de colores, las cuales responden a necesidades derivadas de la experiencia del hablante de aimara. Así, la palabra $q$ 'illu 'amarillo' constituye una red más compleja de vocablos y expresiones cromáticas como chuki q'illu 'amarillo oro', chukiptña 'tendencia al amarillo oro', yuri q'illu 'amarillo similar a la yema de huevo', etc., los cuales presentan una estructuración consistente con la percepción visual. En atención a lo señalado, nuestro trabajo pretende definir las redes de significado que se configuran a través de los vocablos referidos a los nombres de colores. Sostenemos que existen elementos cromáticos centrales de los cuales se proyectan o irradian otras denominaciones que son más periféricas, pero que relacionan de forma integrada, es decir, en estructuras determinadas conceptualmente y motivadas desde la experiencia física.

Palabras clave: redes radiales, aimaras.

\section{ABSTRACT}

Words related to chromaticity are recorded in the Aymara language, many of these names are associated with different color scales, which respond to needs derived from the experience of the Aymara speaker. Thus, the word $q^{\prime} i l l u$ 'yellow' constitutes a more complex network of words and chromatic expressions such as chuki q'illu 'golden yellow', chukiptña 'tendency to golden yellow', yuri q'illu 'yellow similar to egg yolk ', etc., which present a structuring consistent with visual perception. In view of the above, our work aims to define the networks of meaning that are configured through the words referred to the names of colors. We maintain that there are central chromatic elements from which other names are projected or radiated that are more peripheral, but that are related in an integrated way, that is, in conceptually determined structures and motivated from physical experience.

Keywords: radial networks, Aymara.

Forma de citar el artículo (Formato APA):

Huanca, J. (2021). Redes radiales y prototipicidad en los conceptos cromáticos aimaras. Tierra Nuestra. 15(1), 69-83. http://dx.doi.org/10.21704/rtn.v15i1.1405

${ }^{1}$ Universidad Nacional Mayor de San Marcos, Lima, Perú. jhuancaq@unmsm.edu.pe 


\section{Introducción}

Las denominaciones cromáticas, más que un inventario de palabras inconexas, responden a necesidades derivadas de la experiencia del hablante de aimara. Así, la palabra q'illu 'amarillo' constituye una red más compleja de vocablos y expresiones cromáticas como chuki q'illu 'amarillo oro', chukiptña 'tendencia al amarillo oro', yuri q'illu 'amarillo similar a la yema de huevo', etc., los cuales presentan una estructuración consistente con la percepción visual. En atención a lo señalado, nuestro trabajo pretende definir las redes de significado que se configuran a través de los vocablos referidos a los nombres de colores. Sostenemos que existen elementos cromáticos centrales de los cuales se proyectan o irradian otras denominaciones que son más periféricas y que se analizan de forma integrada, es decir, en estructuras determinadas conceptualmente. Nuestro estudio pretende contribuir con el conocimiento de cuáles son las implicancias socioculturales y cognitivas del aimara mediante un estudio que se enmarca en los trabajos de documentación lingüística cuyo objetivo medular es asegurar la vitalidad de las minorías lingüísticas, las cuales evidencian un repliegue considerable, a través de análisis escrupulosos; es menester contar con mayor evidencia de las lenguas minoritarias peruanas actualizadas en contextos reales, pues son los usuarios quienes la explicitan y permiten su revitalización en el caso extremo en que las sociedades soslayen el uso de su lengua materna. Por ello, en principio, son disciplinas heterogéneas las que realizan un abordaje de esta acción, a saber, el análisis del discurso, los enfoques del desarrollo lingüístico, los estudios de fonética experimental, la teoría lingüística propiamente dicha, etc. (Himmelmann, 1988).

Un aspecto importante de la documentación es el uso de técnicas de recolección que posibiliten la obtención de evidencia empírica de alta calidad, la cual puede ser usada para empoderar a los propios usuarios de la lengua (Hale, 1972), además de permitir que el investigador desarrolle las pesquisas con responsabilidad ética en favor de las lenguas en peligro de extinción (Rice, 2006). Por eso nuestro estudio se enmarca en los trabajos actuales de documentación, que requieren de equipos y técnicas de recolección apropiados. En consecuencia, la finalidad fundamental es, además del análisis escrupuloso de evidencia lingüística para la consecución de descripciones exhaustivas de las lenguas naturales cuyo estado es de vulnerabilidad alta, la preservación de estas mediante formatos accesibles (Kritikos, 2015, p. 243). Es importante el trabajo de documentación en aimara y trabajos como el nuestro se corresponde con las necesidades actuales de documentación lingüística, los cuales se comprometen con el trabajo de análisis profundo mediante técnicas de recolección adecuadas (Himmelmann, 2012, p. 187). En nuestro caso, el registro se realizará a través de una grabadora Zoom para realizar un trabajo de elicitación con hablantes nativos $\mathrm{y}$, por supuesto, estos datos podrían derivarse como muestra a otro tipo de estudios (Podesva y Sharma, 2013, p. 5).

\section{Antecedentes}

Entre los estudios considerados como antecedentes de la investigación, estos básicamente son trabajos de descripción gramatical en aimara, aunque también existen estudios acerca de la manifestación cultural de los colores. Cada uno de estos será abordado de manera razonada a continuación, con el objetivo de definir en qué medida se han realizado estudios vinculados con la denominación de colores como producto de la experiencia en los grupos humanos. En tal sentido, será pertinente abordar el estudio en términos amplios a fin de enfocarnos posteriormente en el aimara.

El primer estudio que es pertinente abordar es el trabajo clásico en el ámbito de la antropología: Basic Color Terms: Their Universality and Evolution (1969) de Brent Berlin y Paul Kay. En este, se desarrolla la hipótesis fundamental acerca de la forma en que las denominaciones de colores se estructuran a partir de conceptos básicos. Así, el estudio predice que las denominaciones cromáticas evolucionan en direcciones cuyas restricciones pueden definirse sobre la base del número de conceptos de colores de cada cultura. Así, es posible definir cierta complejidad en algunas culturas, mientras que otras simplifican los conceptos asociados con los colores; sin embargo, es posible determinar intersecciones. El trabajo inicial ha derivado en una ampliación de los elementos recopilados, a través de reajustes metodológicos que devinieron en la comparación de términos culturalmente definidos en megacategorías, las cuales difieren interlingüísticamente (Hardin, 2014, p. 2). Este trabajo 
ha derivado en estudios más acuciosos que proponen planteamientos consistentes con técnicas de análisis sofisticados para recusar el planteamiento fundamental de Berlin y Kay (McCarthy, Winston, Mueller, Watson y Yarowsky, 2019), o para ampliar los resultados iniciales de estos en estudios con resultados que persiguen explicaciones del comportamiento universal de los colores (Loreto, Mukherjee y Tria, 2012), así como descripciones más orientadas al comportamiento particular en lenguas como el árabe por ejemplo (Alrasheed, Al-Mohimeed y Davies, 2013). Aun cuando el debate está abierto, estas referencias serán importantes para definir el comportamiento interlingüístico de los conceptos cromáticos, además de razonar los resultados en lo concerniente al aimara.

Por otro lado, los trabajos sobre el aimara específicamente y, en sentido estricto, sobre el color, están ceñidos a la descripción gramatical. Es importante en este caso el trabajo de recopilación léxica de Felipe Huayhua Pari, quien en su obra Diccionario bilingüe polilectal aimara-castellano, castellano-aimara (2009) presenta un universo léxico importante de la lengua. El tratamiento lingüístico es importante para el reconocimiento de conceptos cromáticos, por ello, esta es una fuente directa que permitirá elaborar el instrumento que permita realizar el trabajo de elicitación posterior. Aunque esta obra no analiza directamente los datos, consideramos que es un punto de inicio por el registro lexicográfico de la lengua, valioso para reconocer los usos actuales y confrontarlos con la evidencia empírica que se recoja del trabajo de campo. Un trabajo adicional del autor constituye un estudio estrictamente gramatical de la lengua (Huayhua, 2001), el cual será analizado a fin de determinar aspectos lingüísticos que puedan derivar al establecimiento de patrones cognitivos vinculados con los conceptos cromáticos en la lengua. Por ello, ambos estudios los consideramos antecedentes de nuestra investigación.

El estudio titulado La estética del color en los tejidos aimaras de la isla de Suriqui de Mario Apaza Apaza (2009) es importante, pues, si bien no se trata de una pesquisa lingüística, ofrece pistas interesantes sobre la disposición de los colores en los tejidos aimaras y sus implicancias culturales, por ello, lo consideramos un antecedente. El autor realiza un análisis estético de los tejidos aimaras. Si bien es cierto el estudio se enfoca en los aspectos artísticos como la disposición de las figuras en los tejidos, que evidencian una compleja simbología; tal es el caso de las irpaq'atas, las cuales presentan una disposición que se configura en términos de una jerarquía armónicamente definida: irpaq'atas mayores, irpaq'atas intermedias e irpaq'atas menores. Estas se reconocen como irpthapitas madres e irpthapitas crías o bebés. Una relación vivencial que se expresan metafóricamente en los tejidos. Este aspecto es importante, pues se pueden evidenciar distintos tipos de conceptualización en las denominaciones de los tejidos; por ejemplo, internamente es posible detectar ciertos espacios en los cuales se disponen figuras animales y que asocian espacios mayores: estos últimos son denominadas con metáforas somáticas como nayrapa 'ojo' o chuymapa 'su corazón'; en otras palabras, se trata de lugares desde los cuales emana la vida. En palabras del autor del estudio: «Este es el lugar donde mana la vida y donde reside la fuerza creadora del arte a través del encuentro de lo sensible con lo racional» (Apaza Apaza, 2006, p. 93). La cita es una prueba de que, más allá de solo la disposición de espacios y colores, los tejidos aimaras en la isla de Suriqui presentan un sentido cultural $\mathrm{y}$, por consiguiente, la disposición y la escogencia de los colores es altamente probable que configure la experiencia de los aimarahablantes y la forma en que estos les confieren un significado particular. En cuanto al uso de colores en los tejidos, estos están regulados por la presencia de un referente importante en la vida de los aimarahablantes: el arcoíris. Las diversas tonalidades de rojo, las diversas tonalidades de verde, entre tantos otras tonalidades de colores, presentan correspondencias lingüísticas específicas en la vida de las tejedoras de la zona, aunque bien podrían corresponderse con cualquier comunidad de habla aimara. Por ello, es importante reconocer qué motivación está implícita en el uso de colores tan variados que funcionan en contraste. Las tejedoras sustentan su creación textil en el reconocimiento de las diversas posibilidades de color que define el arcoíris y, por eso mismo, requieren correspondencias en la lengua. Por ello es menester determinar la carga semántica de las palabras asociadas con el color. Dado que el estudio desarrollado en este subcapítulo es de tipo artístico, será importante, más allá de si las tejedoras usan el color rojo e inmediatamente utilizan un color más claro, en palabras del autor, «[...] k'hantañapataqui (para que produzca luz)» (Apaza Apaza, 2006, pp. 154-155), para determinar la forma en 
que la cromaticidad se configura semánticamente en aimara, a fin de discutir las motivaciones específicas que permiten estructurar la realidad cromática, experimentada visualmente. Notemos que, al igual que en la labor textil se manejan distintas tonalidades de verde, distintas tonalidades de rojo o de cualquier otro color que sirve de herramienta lumínica en los tejidos, el aimara presenta correlatos lingüísticos que sirven para expresar y organizar diversas realidades.

Algunos otros trabajos sobre el aimara serán revisados por tratarse de estudios de gran envergadura sobre la lengua, entre otros, el trabajo de Alfredo Torero cristalizado en Idiomas de los Andes (2002), los estudios escrupulosos de Cerrón Palomino (2000, 2004), entre otros, serán de relevancia para los aspectos gramaticales de la lengua aimara, aunque directamente no se aproximan al fenómeno de las denominaciones cromáticas.

\section{Planteamiento del problema}

Los colores en aimara se expresan en correlatos léxicos que se asocian con realidades que los hablantes reconocen y explicitan en múltiples referentes cromáticos. Uno de estos es khana 'luz', el cual se corresponde con la voz kurmi 'arco iris' o la descomposición en cromatismos diversos que presentan diversas denominaciones (Apaza Apaza, 2009, p. 44). Las denominaciones de colores en la lengua son interesantes porque se manifiestan en actividades como la confección de tejidos, en la cual se reconocen diversas tonalidades que funcionan como clasificadores de la realidad. Entre los múltiples colores detectables en la lengua son detectables las formas chupika 'rojo', qhana chupika 'rojo naranja', paqu chupika 'rojo amarillento, pelirojo', wila 'rojo sangre, rojo violáceo, guinda', wila qispi qala 'rubí, piedra preciosa'. Podría indicarse que se trata de un inventario de vocablos o expresiones en los que se detectan formas lingüísticas que expresan el cromatismo del aimara, el cual podría ser equiparable uno a uno a cualquier lengua natural. Sin embargo, las tonalidades se vinculan con experiencias vividas por los hablantes de aimara, entre las cuales es posible mencionar actividades como la confección textil, la elaboración de cerámicas, entre otras labores. A propósito de los diferentes referentes de colores, se formulan las siguientes preguntas que enfrenta nuestra investigación:
3.1. ¿De qué manera se organizan los cromatismos en la lengua aimara?

3.2. ¿En qué medida estas estructuras permiten reconocer procesos de organización de la realidad que se inicia con la percepción visual?

3.3. ¿Cuáles son las motivaciones específicas que están involucradas en los mecanismos de estructuración de la realidad cromática en aimara?

\section{Hipótesis}

Los cromatismos en aimara se configuran en redes radiales de significados cuya motivación se vincula con las actividades cotidianas de los hablantes, las cuales involucran una necesaria organización de la realidad percibida visualmente.

\section{Objetivos}

\subsection{Objetivo general}

Determinar las redes de significados que están involucradas en la organización de los cromatismos en aimara.

\subsection{Objetivos específicos}

a. Determinar las motivaciones que determinan la organización de los conceptos cromáticos en aimara.

b. Reconocer la forma en que están organizadas las estructuras de significado relativos a conceptos cromáticos en aimara.

\section{Metodología}

El estudio se sustenta en la recopilación de unidades léxicas en las que se reconozca la experiencia del aimarahablante respecto del color. Para ello, se consultarán fuentes lexicográficas y se procederá a la estructuración de una entrevista para ampliar los datos obtenidos.

\subsection{Tipo y diseño de investigación}

Nuestro estudio se enmarca en los trabajos de tipo descriptivo-explicativo. En suma, el trabajo se corresponde con un diseño de carácter transeccional y descriptivo, dado que se recolectará evidencia de conceptos cromáticos en aimara en un periodo específico a fin de describir y explicar en qué consisten los mecanismos conceptuales que organizan y estructuran los significados de tipo cromático en aimara. 


\subsection{Unidad de análisis}

Los conceptos de tipo cromático y las estructuras conceptuales cromáticas motivadas en aimara

\subsection{Muestra}

La muestra está constituida por vocablos de la lengua aimara asociados con el color. Estos datos serán recopilados a través de entrevistas directas realizadas en las provincias de Moho y Huancané (Puno). Ambas zonas son de habla aimara, de manera que será fundamental realizar el trabajo de campo. Consideramos que el número de hablantes idóneo es cuatro hombres y cuatro mujeres, y los datos que se recopilarán serán de alta calidad, a través de una grabadora Zoom $\mathrm{H} 4$.

\subsection{Técnicas de recolección de datos}

Para obtener evidencia empírica de conceptos cromáticos en la lengua aimara se usarán dos técnicas de recolección: la primera de ellas consiste en un muestreo a través de la elicitación de palabras; sin embargo, también recogeremos evidencia acústica espontánea en la que se documentarán los conceptos cromáticos en contextos de interacción discursiva en las zonas de habla aimara contempladas en la subsección de la muestra.

\section{a) Elicitación de conceptos cromáticos en aimara.} Esta técnica consiste en indicarle una expresión en castellano con la finalidad de que los hablantes de aimara la reiteren en su lengua materna. Estas emisiones serán registradas a través de una grabadora digital Zoom, modelo $\mathrm{H} 4$, que permite obtener datos de alta calidad. Con ello cumpliremos con los requerimientos técnicos que amerita una investigación de esta envergadura.

b) Documentación de eventos comunicativos espontáneos. La labor adicional para recoger datos será coparticipar de la entrevista a través de la colaboración participante con hablantes de la zona. Nuestro conocimiento de la lengua como nativohablante permitirá obtener audios en contextos de mayor libertad e interacción.

\section{Marco teórico}

\subsection{El papel de la corporeización}

La Lingüística cognitiva presenta entre sus principales hipótesis que es la experiencia cotidiana la que define la forma en que conceptualizamos la realidad. Además, este marco de experiencias está canalizado por la percepción inmediata del mundo físico, de manera que el eje que define el sistema conceptual humano y, por lo tanto, también el lingüístico. El concepto de corporeización que desarrollamos en este subcapítulo, presenta una deuda directa con el trabajo de Rohrer (2013, pp. 25-47). Si partimos de la afirmación de que el lenguaje humano constituye un continuo con la cognición general, la corporeización o fenómeno discutido por Rohrer es un mecanismo mediante el cual determina el sistema conceptual y, por supuesto, orienta la estructuración del significado. A fin de despejar dudas sobre las diversas interpretaciones que ha originado este concepto, el autor se plantea la tarea inicial de definir los doce sentidos que se derivan del concepto de corporeización, de tal manera que inicia con la primera aproximación a este, propuesta por Lakoff y Johnson (1980), en la cual se asume a la corporeización a través de la direccionalidad presente en la metáfora conceptual; es decir, del dominio de origen las proyecciones se dirigen hacia el dominio meta. Por lo tanto, el uso del concepto de corporeización, inicialmente estuvo subordinado al de metáfora; sin embargo, como el mismo autor sostiene más adelante, este concepto tomó forma a través de reflexiones profundas y estudios minuciosos que, incluso, se respaldan en los avances en neurociencias. Otra de las acepciones adicionales que resulta interesante sobre la corporeización es la de contexto sociocultural, en la cual se ubican no solo el cuerpo como entidad que experimenta directamente la realidad, sino la mente y el lenguaje mismo como parte de la cognición general; además, se puede entender la corporeización como el conjunto de cambios que experimenta el ser humano desde que es huevo o cigoto y se configura en un feto hasta el proceso de cambio de niño a adulto, por ello se estudian estos cambios en cuanto a la adquisición de ciertos términos espaciales; igual de importante es el sentido de corporeización entendido como los cambios evolutivos; algunos otros sentidos potenciales amplían la complejidad y la multivocidad de este concepto. Basado en esta primera aproximación al concepto, el autor realiza un recuento histórico de esta noción a fin de definir cuál ha sido el camino de reajuste y la forma en que actualmente se asume el concepto de corporeización, ya no como un 
concepto subordinado, sino como el núcleo teórico que explica la cognición. Es importante destacar que la corporeización desde que fue usado por primera vez en el seno de la Lingüística Cognitiva, estuvo ligado a la aclaración de los relictos corporales detectables en el lenguaje humano.

La propuesta sobre la corporeización hacia la década del 80 se utilizó para explicar el lenguaje en términos más ambiciosos: las pesquisas, desde la fisiología, la interacción que ocurre entre el organismo y el medio ambiente, procuraban arribar a resultados consistentes sobre una cantidad mayor de fenómenos lingüísticos. La dependencia respecto de la metáfora conceptual le dio lugar a un enfoque en el que la corporeización se apoya en los resultados de las ciencias cognitivas. En tal sentido, más que solo una noción dependiente, las tendencias más recientes sostienen la presencia de una mente corporeizada; esto supone un cambio radical en el cual se sostiene que la mente además de operar en lo concerniente a operaciones como el movimiento o la percepción sensorial, juega un papel importante en la conceptualización. En tal sentido, percepción y conceptualización se abordan en confluencia a través de esta mente corporeizada. En la actualidad, es necesario un estudio interdisciplinario para definir la real magnitud de la corporeización. La propuesta, en palabras del autor, necesita de avances más sólidos en los cuales se asocien los resultados en disciplinas diversas como la sociología, la psicología, la antropología y las neurociencias, pues la exigencia sobre cómo la corporeización experiencial o la corporeización fisiológica están involucradas en el lenguaje, además de determinar de qué manera ocurre esta relación. El campo de estudio es promisorio.

\subsection{Las redes radiales de significado}

En un interesante trabajo sobre polisemia, prototipos y categorías radiales, aborda un fenómeno vinculado con cualquier palabra en las lenguas, su variedad de significados. Barbara Lewandowska-Tomaszczyk (2007) parte de la determinación de cuatro propiedades que definen el análisis de la polisemia en el marco de la semántica cognitiva. Toda palabra es polisémica y, aun cuando se piense que su distinción principal con la homonimia es el rastreo histórico de vínculos etimológicos, los recursos tradicionales son insuficientes para realizar una explicación de qué define la ocurrencia de diversos significados. Al inicio, la autora considera que un rasgo de la polisemia es la flexibilidad del significado, el cual puede definirse como la proximidad de significados entre palabras que presentan igual forma pero etimologías diferentes y el alejamiento de significados cuyo origen es el mismo. Por otro lado, la organización de estructuras radiales permite explicar de qué manera se aparece la polisemia de las palabras pues todo gira en torno a un elemento central que configura relaciones estructuradas de significados. Además de ello, y vinculado con lo anterior, es posible determinar que existen elementos prototípicos que sirven como el centro del cual irradian los demás significados. Un último elemento que la autora propone es el de las redes esquemáticas, las cuales definen varios centros estructurados y no solo uno. La polisemia como fenómeno ecuménico se explica entonces no como un solo significado que se expresa a través de diferentes usos en situaciones comunicativas concretas, sino que se trata de múltiples significados estructurados en un eje que es la categoría prototípica que presenta atributos básicos que los demás miembros de la red radial comparten. Es importante señalar que la autora desarrolla la propuesta de autores como Lakoff, Langacker, entre otros, con la finalidad de proponer un desarrollo de la teoría de prototipos en consonancia con una explicación de la polisemia a partir de los presupuestos conceptuales de un elemento nuclear que dirige la aparición de nuevos sentidos o significados.

Un aspecto interesante de la propuesta es la relación sólida que se genera a través del prototipo, pues las categorías son una forma de conceptualizar la realidad, a partir de elementos que son familiares y que presentan una fuerte motivación. No olvidemos que la teoría de prototipos asume que, si bien los elementos periféricos carecen de capacidad predictiva están motivados, esto es, no son arbitrarios. Asimismo, las nuevas tendencias permiten asegurar un camino de reflexión y discusión sobre la propia teoría de los prototipos. La autora plantea que la versión extendida hace posible pensar en efectos prototípicos que aseguran una explicación más amplia de la polisemia, pues esta puede vincular categoría de forma más flexible. Así, el ejemplo de avión que puede expresarse a través del concepto de pájaro es el ejemplo más visible. La red de esquemas que autores como Langacker propone no es sino la consideración de estructuras más complejas y de mayor 
alcance, en la cual las categorías como formas de organizar la realidad a través de los significados presentan varios centros que irradian significados en subconjuntos de elementos categoriales. Un aspecto interesante de este enfoque es que reorienta al centro del debate la polisemia, un concepto que el siglo pasado fue abordado de manera inadecuada o simplemente se lo soslayó. Por eso es importante rastrear la naturaleza de esas relaciones. Autores como Lakoff, por ejemplo, proponen la existencia de modelos cognitivos idealizados, a fin de determinar cómo la categorización hace posible la polisemia nuevamente en torno de un elemento más relevante. Es de esta forma que el significado se aborda de forma conjunta con los diferentes modelos cognitivos idealizados, entre los que Lakoff destaca la metáfora y la metonimia. Existe un campo promisorio de acción y es importante que los estudios definan qué propiedades medibles presenta la red radial y sus múltiples significados que, además, opera en torno a subcomponentes centrales. Los experimentos en neurociencias podrían dar algunas pistas promisorias de los elementos en cuestión.

\subsection{La integración conceptual}

El concepto de integración conceptual que desarrollaremos se sustenta en la exposición de Mark Turner (2007). Esta se define como una operación mental básica que permite vincular espacios mentales, incluso si estos son muy diferentes. Es posible que una acción en concreto como la exposición de un trabajo genere un conjunto de imágenes previas que confluyen en un espacio mental específico, el cual, en determinado momento, se asocia con un espacio mental diferente, esta vez conformado por imágenes que se corresponden con la declaración de amor a una compañera de colegio. Los casos anteriores son una clara muestra de la capacidad humana de establecer relaciones entre imágenes, organizadas en espacios mentales muy distintos, sin que se generen confusiones o sinsentidos: la integración conceptual es producto mismo de la larga historia evolutiva y es la que, en última instancia, hace posible la conformación de nuestra propia naturaleza. El autor, respecto de ello, propone una serie de principios que definen su constitución: la vinculación parcial entre elementos significativos de los espacios mentales, la existencia de un espacio mental genérico que sirve de puente relacional, la existencia de un espacio mental integrado en el que entonces es posible vincular imágenes relativas a situaciones muy distintas, y, finalmente, la proyección que restringe selectivamente algunos elementos de los espacios para la meta final que es la integración.

La emergencia de otros espacios mentales integrados nuevos se sustenta en la existencia de otros que han sido conformados previamente, y opera a través de tres direcciones: composición, finalización y elaboración. La primera de ellos se enmarca en los elementos de entrada de un espacio en particular, la segunda de ellos integra elementos de espacios divergentes, mientras que la última consiste en la ejecución de integraciones como simulaciones. Esta integración conceptual, además de los principios previos indicados, opera a partir de otros que funcionan como directrices específicas relativas a lo que Turner denomina relaciones vitales. El primer principio es topológico, es decir, se vincula con lo espacial y define similitudes esperables entre objetos. El segundo principio consiste en la conformación de patrones en términos más amplios, es decir, no se enfoca específicamente en elementos espaciales, la semejanza o similitud entonces permite establecer elementos integrados conceptualmente. El principio de integración consiste en el logro mismo de la integración. Además, el cuarto principio maximiza los espacios vitales en la integración (estos son experienciales); el principio de intensificación de relaciones vitales las enfatizan dramáticamente; el principio web asegura que las conexiones sean pertinentes; el principio de desembalaje involucra la reconstrucción de toda la red de relaciones; finalmente, los principios de relevancia y de comprensión involucran, por un lado, se decantan por la pertinencia y la importancia de los espacios que deben integrarse, y por la integración de espacios comprimidos respectivamente.

La relevancia de la integración conceptual en términos lingüísticos radica en que esto es lo que nos diferencia de otras especies animales. La posibilidad de establecer relaciones entre espacios mentales involucra mecanismos de tipo conceptual que hace posible trabajar con esquemas previos instalados para estructurar las experiencias nuevas a partir de los principios reguladores descritos previamente, que actúan sobre elementos gramaticales de distinto tipo como los adjetivos, los nombres, y cualquier otra unidad lingüística. Visto de esta forma, el autor plantea un mecanismo integrador complejo: la integración 
conceptual de doble alcance. Este tipo de integración, para el cual los seres humanos estamos especialmente adaptados, y es el lenguaje el que hace posible que este ocurra, nos define, y puede resultar un mecanismo casi inconsciente, dado que opera de forma rutinaria. Así, si bien es cierto, los monos vervet pueden ser capaces de lograr emisiones para fines específicos como alertar acerca de la presencia de algún depredador que pudiera atentar contra la vida de algún miembro de la especie (es decir, se usa para una situación específica en concreto), esta estrategia parece no llegar muy lejos y el adiestramiento de los chimpancés es revelador al respecto (el aprendizaje de elementos léxicos, si bien puede ser profuso es en términos de su uso bastante limitado); esto da pistas sobre la complejidad del lenguaje humano que usa estos mecanismos de integración para cualquier situación posible, es decir, carece de correferencia específica, incluso serviría para analizar, impugnar o explicitar cualquier escenario potencial, es decir, que puede ser posible de ocurrir (o que incluso haya ocurrido ya). La evidencia entonces parece ser consistente acerca de la naturaleza misma de la integración conceptual como determinante para nosotros como especie.

\section{Discusión y análisis}

Iniciaremos la discusión y el análisis de los datos presentado los aspectos básicos de los correlatos léxicos asociados a los colores en aimara. En la lengua se presentan tres elementos nominales relativos a los colores primarios. Estos son básicamente chupika 'rojo', q 'illu 'amarillo' y larama 'azul'. Por otro lado, los colores secundarios son laranja q illu 'anaranjado', ch'uxña 'verde' y ch'imaxa 'violeta'. Esta correspondencia básica es importante para las relaciones posteriores entre formas nominales $o$ verbales que expresan la cromaticidad propia de la cultura aimara, la cual, como se verá luego, es aplicable en actividades de diverso tipo como la confección textil, entre otras.

La base léxica cromática genera núcleos de significado en la lengua que amerita un análisis apropiado respecto de los efectos y las relaciones con categorías que se desprenden de la actividad y la experiencia propia del hablante de aimara. Analizaremos en principio la forma en que se organizan las redes de significado en el que la forma base es un concepto cromático primario

\subsection{Tonos de colores primarios}

Iniciamos el análisis de datos con la forma en que las denominaciones de colores primarios involucran la conformación de categorías semánticas estructuradas y definidas a través de la percepción de la realidad cromática.
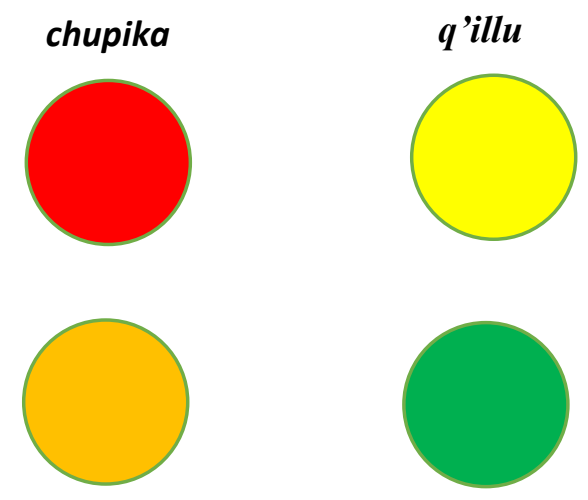

laranka q'illu ch'uxña

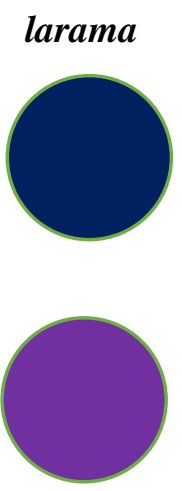

ch'imaxa

Figura 1. Colores primarios y secundarios en aimara 


\subsubsection{Tonos relativos a chupika}

Este color se relaciona conceptualmente con diversas referentes; además, existen otros conceptos relacionados con el rojo. Así, chupika 'rojo' se relaciona directamente con las formas qhana chupika 'rojo naranja' у paqu chupika 'rojo amarillento o pelirrojo'. Sin embargo, es posible que la forma phaq'u 'cabello rojo bermejo'; y esta forma a su vez se vincula con phaq'u wila 'rojo sangre, rojo violáceo, guinda' (de wila 'sangre' y phaq'u 'rojo bermejo'); finalmente, la forma wila qispi qala 'rubí, piedra preciosa' incluye la palabra wila 'sangre' y qala 'roca'.

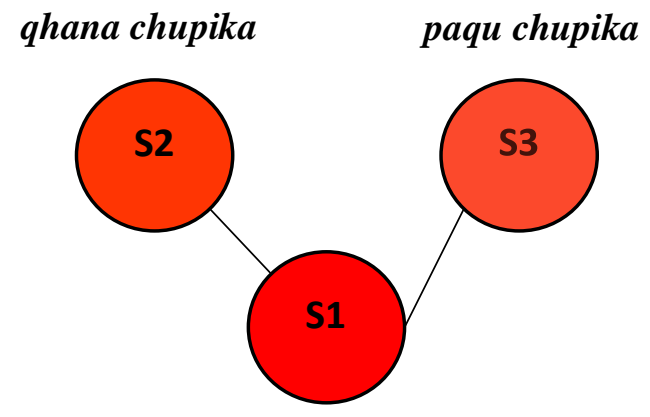

chupika
En principio, los conceptos cromáticos de tonalidad en rojo se asocian con la degradación de la intensidad. En tal sentido, el rojo naranja y el rojo amarillento presentan menos intensidad que el rojo propiamente dicho. Esta realidad puede extenderse a categorías de orden físico como el color de cabello o ciertos referentes que se aproximan cromáticamente a ciertas tonalidades. Tal es el caso de wila qispi qala 'rubí, piedra preciosa color sangre'. La motivación de estos conceptos cromáticos es física: el aimarahablante establece categorías sustentadas en la gradación cromática de acuerdo con la percepción física y la forma en que este experimenta el color.

Figura 2. Redes semánticas en torno a chupika y phaq'u wila en aimara

\subsubsection{Tonos relativos a q'illu}

En torno del nombre $q$ 'illu se organiza una red bastante compleja de tonalidades cuya motivación es, en efecto, eminentemente de orden físico. Los datos brindan una muestra reveladora sobre cuáles son los elementos experienciales que se categorizan en la lengua y, por supuesto, determinan relaciones semánticas en las cuales se constata la conformación de recursos de orden léxico; es decir, los conceptos cromáticos se relacionan con estructuras conformadas por más de un constituyente, además de mecanismos morfológicos. Por ejemplo, algunas de las formas son chuki q'illu 'amarillo oro', chukiptña 'tendencia al amarillo oro' yuri q'illu 'amarillo yema de huevo', churi q'illu 'amarillo bajo', q'illukiptaña 'volverse amarillo', anti q'illu 'amarillo claro' y tunqu q'illu 'amarillo maíz'. Estos elementos, semánticamente, se proyectan del núcleo q'illu y se dispersan de acuerdo con las tonalidades de amarillo que, producto de la experiencia, se ajustan a la percepción visual de tipo tonal en relación con el color.

De acuerdo con la figura 3, los conceptos cromáticos giran en torno a q'illu, dado que este es el significado central o prototípico. De este concepto central se proyectan otros significados que se refieren a elementos físicos, ya sean objetos comestibles como el maíz, el huevo o el oro. Respecto de este último, incluso se proyecta la forma derivada chuquiptña 'tendencia al amarillo oro'. También puede aludir a diferentes tipos de tonalidad del amarillo propiamente dicho. Ello supone que, ya sea a través de la percepción directa de los colores o la motivación a través de entidades específicas de la realidad, la cromaticidad asociada con el amarillo dista de ser simple. Además de los datos presentados previamente, es posible detectar otros componentes léxicos que hacen referencia al amarillo en términos conceptuales cuando la tonalidad está vinculada con el cabello. De esta forma, es posible 
detectar denominaciones cromáticas como paqu 'amarillo dorado, cuando se refiere al pelo', ch 'umpi 'amarillo café, cuando se refiere al pelo', millu 'amarillo beiges, cuando se refiere al pelo'. El siguiente concepto cromático que analizaremos será el de larama 'color primario azul'.

\subsubsection{Tonos relativos a larama}

El color primario larama también se constituye en el significado central de diversas realidades conceptualizadas y categorizadas por los hablantes de aimara. Aunque se trata de elementos léxicos diferentes, semánticamente se asocia con sajuna 'azul cielo', laqhampa 'azulino', laramachaña 'azul cielo', laramaptaña 'volverse azul', quуи 'azul violeta', ch 'iyara larama 'azul marino', ch 'uxña larama 'azul verdoso', qhusi 'celeste, referido al ojo' y laqampu 'celeste'. En apariencia los vocablos pueden parecer carentes de relación, pero, más allá de las divergencias en la forma (laramachaña respecto de sajuna son diferentes desde el punto de vista fonológico), la relación la debemos buscar a nivel conceptual o semántico. En tal sentido, las categorías léxicosemánticas necesariamente implican una relación profunda a nivel conceptual. Por ello, proponemos la siguiente red radial de significados asociados a larama.

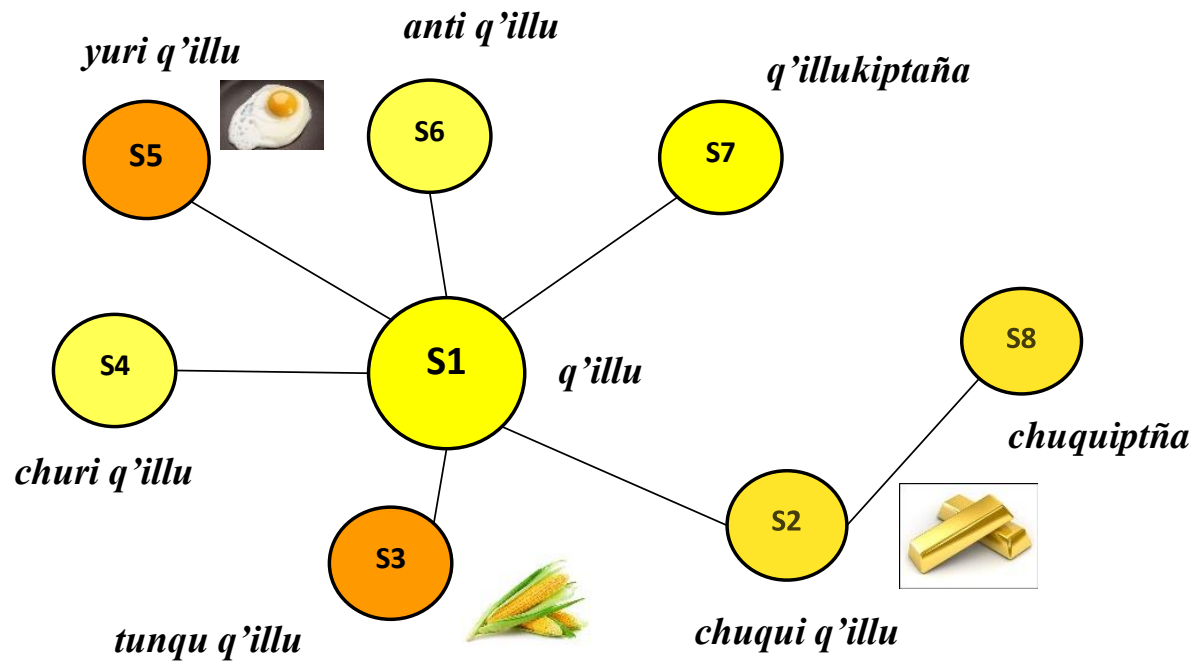

Figura 3. Red radial del concepto cromático q'illu en aimara

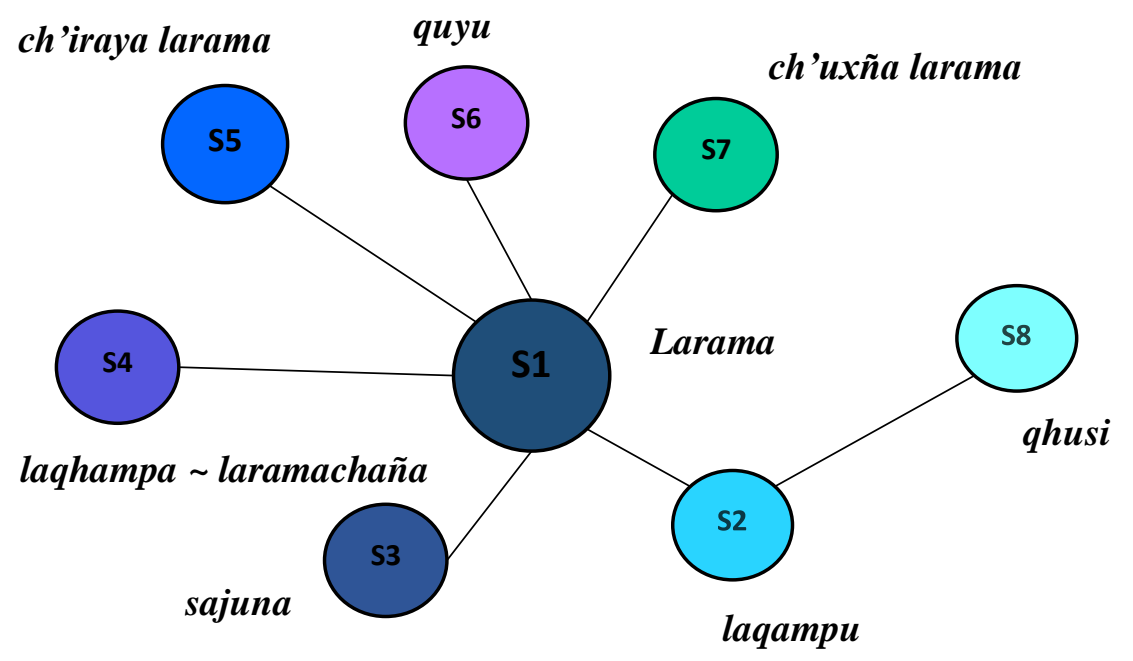

Figura 4. Red semántica relativa a larama en aimara 
Los conceptos asociados a larama 'azul' presentan una dispersión bastante definida en términos semánticos. La variabilidad tonal del azul es parte de la experiencia cromática conceptualizada por los hablantes. Notemos que existen ciertas motivaciones visuales relacionadas con el entorno del hablante aimara: el cielo, el color del lago, el ojo cuando está afectado por la catarata, etc. Estas distinciones son interesantes porque demuestran que culturalmente es posible que las categorías que se conforman sean heterogéneas. En comparación con otras lenguas en las cuales el azul presenta escasas variaciones, el aimara organiza la realidad a través de categorías de variaciones mínimas de azul. Con ello, los colores primarios son fuente de tonalidades que se complementan y que, incluso, podrían representar simbólicamente la forma en que se crean productos culturales como los tejidos. La complejidad semántica de la forma en que el hablante aimara organiza la realidad cromática también se extiende a los colores secundarios. Estos se analizarán en el siguiente subcapítulo.

\subsection{Tonos de colores secundarios}

La correspondencia de conceptos cromáticos con redes de significados diversificados es evidente en la conformación de categorías asociadas con colores secundarios. Abordaremos los tres más relevantes, de menor a mayor grado de complejidad semántica.

\subsubsection{Tonos cromáticos relacionados con laranja}

Laranja se aproxima cromáticamente a q'illu 'amarillo': una de las tonalidades del amarillo se expresa mediante la forma laranja q'illu 'amarillo naranja'. Pitu churi 'naranja bajo' hace referencia, mediante un elemento léxico relativo al espacio, esto es, churi 'bajo', la tonalidad menos intensa de laranja. La correspondencia con la realidad percibida visualmente y la aplicación de una tonalidad específica en la confección de polleras se expresa en el vocablo sisira 'naranja usado en las polleras'; de este se deriva la forma sisira yarita 'flor de una planta de color naranja (en la tonalidad propia de sisira); finalmente, la forma wantura 'anaranjado bermellón' es otra de las tonalidades que se incluyen en la red de significados cromáticos de laranja. Un caso adicional que debe incluirse en el análisis de esta categoría cromática es el que se corresponde con el color primario chupika. En tal sentido la forma nominal qhana chupika 'rojo anaranjado' es otra de las categorías que se intersectan en la escala cromática de naranja respecto de los colores primarios. En otros términos, las redes radiales podrían implicar intersecciones entre formas intermedias constituidas por los colores secundarios y sus respectivos conceptos cromáticos en aimara.

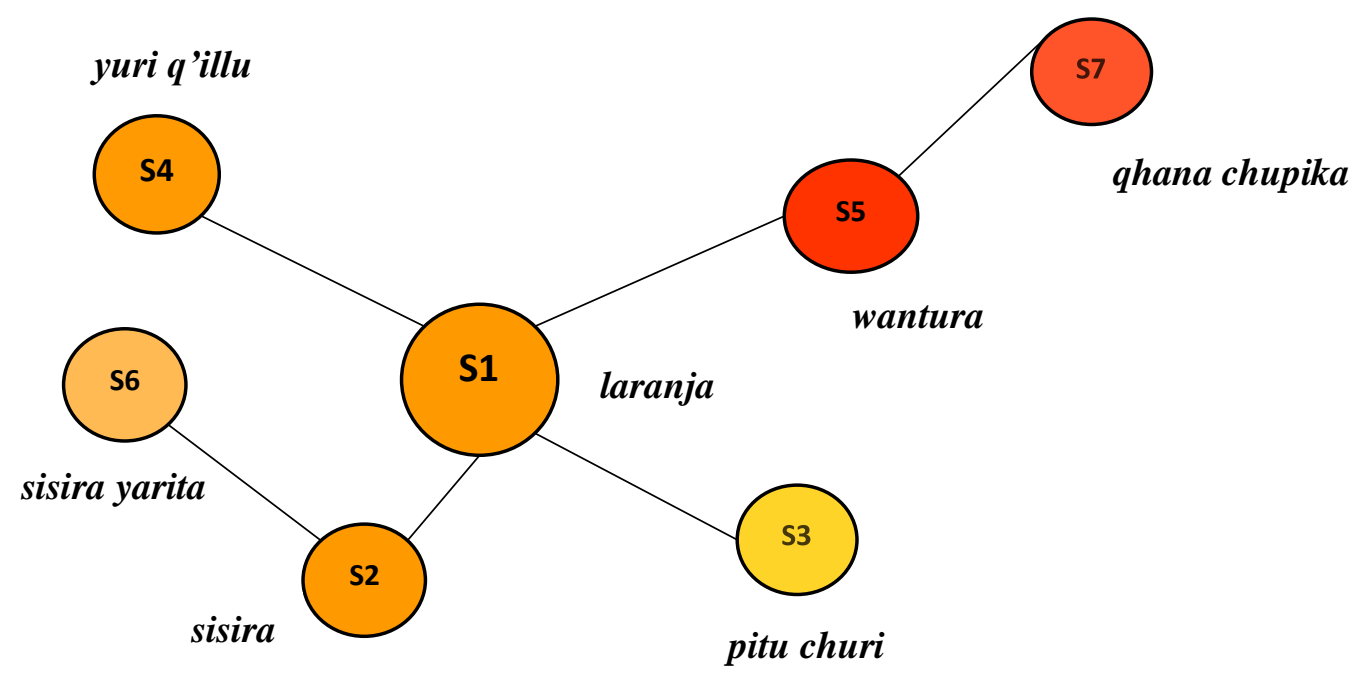

Figura 5. Red semántica del concepto cromático laranja en aimara 


\subsubsection{Tonos cromáticos relacionados con $\boldsymbol{c h}$ 'uxña}

En cuanto a ch'uxña 'verde', la red semántica es ciertamente más compleja e incluye tonalidades mucho más heterogéneas. Uno de los casos más notorios es la inclusión del verde fosforescente qupa ch'uxña que se vincula con la forma verbal qupachaña 'dar de verde, verdear'; esta última semánticamente conforma un bloque con qupachasiña 'verdeguear, comenzar a perderse el verde', qupachanuqaña 'ponerse verde el campo con los sembrados' y qupanchaña o qupchaña 'dar tono de verde' (que podría relacionarse con qupachaña). Algunos casos adicionales de la red semántica están conformados por ch'uxña umiña o qupa: 'esmeralda', $k$ 'intu 'hierba y otras plantas, hojas muy verdes, verde oscuro intenso', muri-llullu 'verde por madurar, verde pasto tierno', qhusi 'verde azulado' y ch 'uxña qispi qala 'piedra verde esmeralda'. Como se aprecia en los datos, los conceptos cromáticos están motivados por el reconocimiento y la organización conceptual de elementos del entorno, sustancialmente plantas. Eso supone el reconocimiento de diferentes matices de verde relacionados con procesos (maduración, apogeo, deterioro). La red semántica es la que sigue.
La red integra conceptos cromáticos nominales y verbales. Así. Ch'uxña incluye no solo tonalidades que devienen de la percepción del color, sino de la relación entre tonalidades y proceso específicos relacionados con la vegetación. Por ejemplo, el verbo qupachaña 'dar de verde, verdear' está directamente relacionado con muri-llullu, pues se refiere al inicio de la coloración verde en el campo. Sin embargo, esta tonalidad no es permanente, pues el pasto seco supone la pérdida de color; por ello, existe la denominación verbal qupachasiña para referirse a un tipo de verde menos intenso. También se detecta el color verde asociado con una piedra preciosa (la esmeralda), se trataría de un mecanismo metonímico que asegura la correspondencia léxica a través de esta categoría motivada por el contacto directo con el mineral que se desplaza por el color para la delimitación del concepto cromático. El último elemento que analizaremos es el de ch'imaxa 'violeta', el cual también presenta diversas denominaciones que suponen la conceptualización de categorías más específicas que solo el patrón genérico que expresa el color violeta.

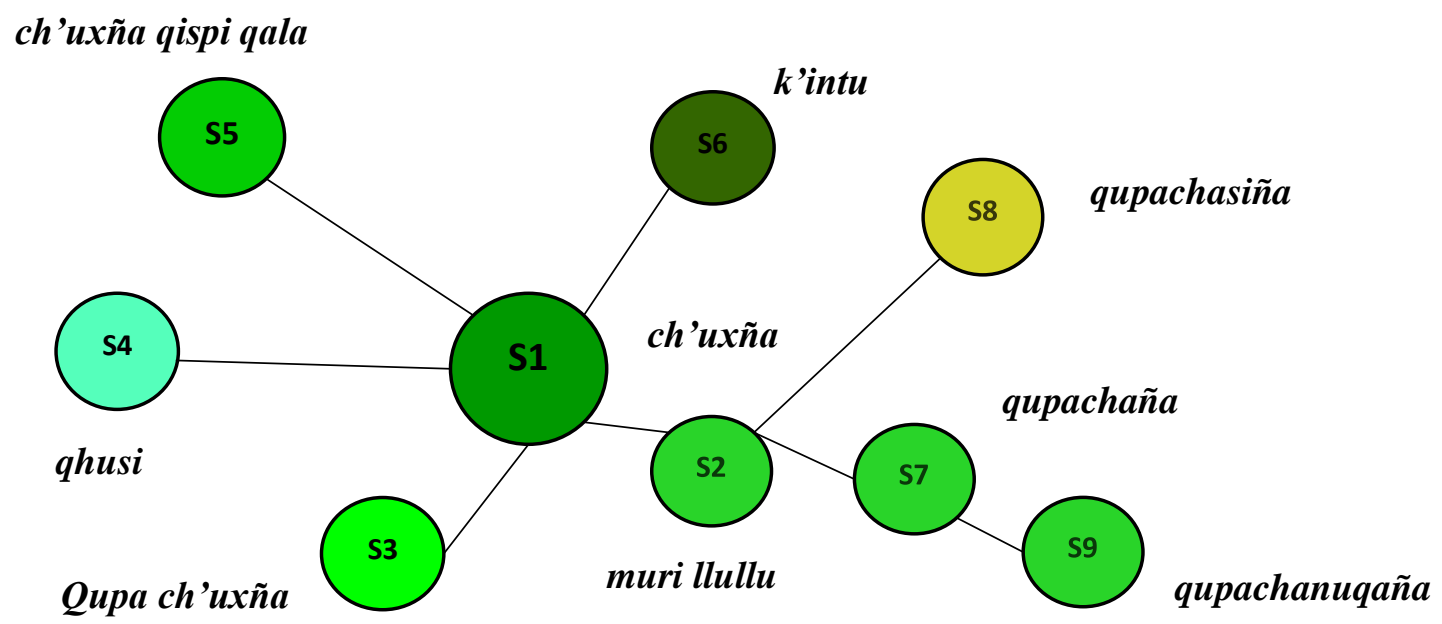

Figura 6. Red semántica de ch'uxña en aimara 


\subsubsection{Tonos cromáticos relacionados con ch'imaxa}

El concepto cromático ch 'imaxa 'violeta' también es parte de los mecanismos conceptuales que categorizan las experiencias del aimarahablante para ordenar realidades más allá de lo cromático. Como en los demás casos, hemos detectado una serie de elementos nominales y verbales que conforman una red menos profusa que la de ch'uxña pero que ofrece evidencia empírica sobre la organización de los cromatismos sustentados en la experiencia y su correspondiente estructuración a través del lenguaje. Las categorías pueden resultar inespecíficas en el sentido de que suponen ciertas transiciones al color pleno; por ejemplo ch 'imxaru jaxsuña 'tendencia a morado' revela la experiencia tonal de aproximación al concepto cromático pleno, pero se le considera parte de la familia de conceptos que presenta como núcleo prototípico la forma ch'imaxa. Por otro lado, la experiencia de eventos que afectan el físico de un individuo como el golpe y sus consecuencias en la coloración de la piel se expresa en forma como q'üta 'amoreteado, partes acardelanados con golpes', que refleja la conceptualización del padecimiento físico en torno del color. Un caso más que se corresponde con el anterior es el de jusqullu 'amoreteado, cardenal de golpe'. Por otro lado, la aproximación al morado se expresa en la construcción verbal larama jusqulluchaña 'hacer morado', en la cual se configura la idea de proceso a través del cual el concepto cromático central aparece. $\mathrm{Si}$ se trata de la conservación del color en un tiempo determinado, este se expresa a través de la forma verbal larama jusqulluchata 'tener amorotado'; finalmente, significados más periféricos son los que presentan cierta tonalidad de color pero evidencian la predominancia del concepto cromático primario, es el caso de quyu 'azul violeta, cardenal' o $k^{\prime}$ ulli 'añil', en los cuales predomina el color azul, aunque presentan cierta tendencia cromática que los asemeja al violeta. La red semántica del vocablo en cuestión se propone a continuación.

La red radial de ch'imaxa en aimara irradia tanto procesos como categorías semántico-nominales. Esta red cuenta con elementos periféricos o no prototípicos: quyu y k’ulli, pues son conceptos cromáticos más próximos a la experiencia perceptual con el azul.

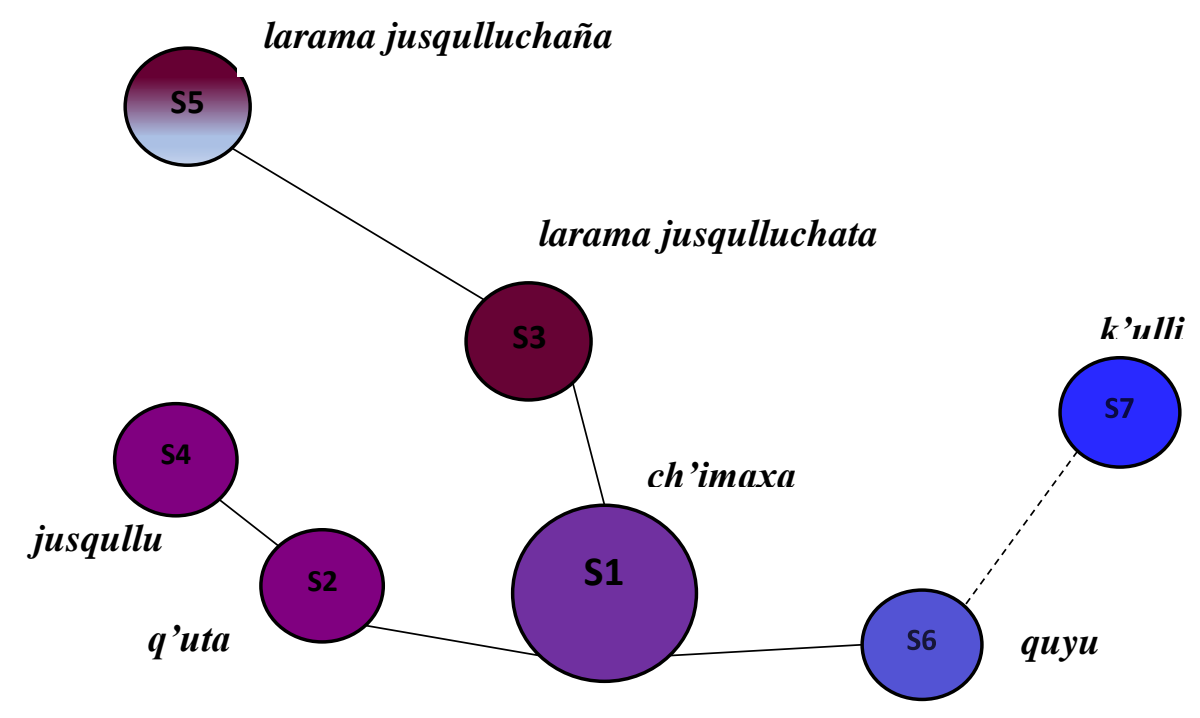

Figura 7. Red semántica del concepto cromático ch'imaxa en aimara 


\section{Conclusiones}

- En cada una de las redes semánticas propuestas para el aimara, constatamos la presencia de mecanismos perceptuales que derivan en la conceptualización de categorías de colores bastante precisas.

- Estas categorías organizan la experiencia cromática de los hablantes aimaras y permiten estructurar los significados de tal forma que se asocian con la realidad, ya sea la forma de disponer los colores en actividades como la confección de prendas, las labores agrícolas, el reconocimiento de minerales, entre otros.

- Los conceptos cromáticos están estructurados y organizados de manera que funcionan como mecanismos de ordenamiento de la realidad física de tipo cromático, la cual se experimenta visualmente.

\section{Conflictos de intereses}

Los autores firmantes del presente trabajo de investigación declaran no tener ningún potencial conflicto de interés personal o económico con otras personas $\mathrm{u}$ organizaciones que puedan influir indebidamente con el presente manuscrito.

\section{Contribuciones de los autores}

Preparación y ejecución; Desarrollo de la metodología; Concepción y diseño; Edición del artículo; Supervisión del estudio: JH.

\section{Bibliografía}

Al-rasheed, A. S.; Al-Mohimeed, N. S. y Davies, I. R. L. (2013). Berlin and Kay's Theory of Color Universals and Linguistic Relativity: The Case of Arabic. Journal of Modern Education Review, 3(5), 366-386.

Berlin, B. y Key, P. (1969). Basic Color Terms: Their Universality and Evolution. Berkeley: University of California Press.

Cerrón P., R. (2004). El aimara como lengua oficial de los incas. Boletín de Arqueología PUCP, 8, 921.

(2000). Lingüística aimara. Cuzco: C.E.R.A. "Bartolomé de Las Casas".
Kritikos, E. (2015). Applying Current Methods in Documentary Linguistics in the Documentation of Endangered Languages: A Case Study on Fieldwork in Arvanitic. Athens Journal of Philology, 2/4, 243-254.

Hale, K. (1972). Some questions about anthropological linguistics: the role of native knowledge. In Dell Hymes, ed. Reinventing Anthropology. New York: Random House, 382-397.

Hardin, C. L. (2014). Berlin and Kay Theory. Encyclopedia of Color Science and Technology, 1-4.

Hardin, C. L. y Maffi, L. (edit.) (1997). Color categories in thought and language. Cambridge: Cambridge University Press.

Himmelmann, N. P. (2012). Linguistic Data Types and the Interface between Language Documentation and Description. Language Documentation \& Conservation, 6, 187-207.

(1998). Documentary and descriptive linguistics. Linguistics, 6, 161-195.

Huayhua P., F. (2001). Gramática descriptiva de la lengua aimara (Aymara aru yatiwi). Lima: Negocios Arco Iris S. R. L.

Huayhua P., F. (2009). Diccionario bilingüe polilectal aimara-castellano, castellano-aimara. Lima: Fondo Editorial UNMSM.

Lakoff, G. y Johnson, M. (1980). Metaphors We Live By. Chicago: The University of Chicago Press.

Lewandowska-Tomaszczyk, B. (2007). Polysemy, Prototypes, and Radial Categories. En Dirk Geeraerts y Hubert Cuyckens (Eds.). The Oxford Handbook of Cognitive Linguistics, pp. 139-169. Oxford: Oxford University Press.

Loreto, V.; Mukherjee, A. y Tria, F. (2012). On the origin of the hierarchy of color names. Proceedings of the National Academy of Sciences May 2012, 109 (18) 68196824; DOI: 10.1073/pnas.1113347109.

McCarthy, A.; Winston, W.; Mueller, A.; Watson, B. and Yarowsky, D. (2019). Modeling Color Terminology Across Thousands of Languages. Proceedings of the 2019 Conference on Empirical Methods in Natural Language Processing and the 9th International Joint Conference on Natural Language Processing, 2241-2250, Hong Kong: China.

Moseley, C. (ed.). (2010). Atlas of the World's Languages in Danger, 3rd edn. Paris: Unesco 
Publishing. Online version: http://www.unesco.org/culture/en/endan geredlanguages/atlas

Podesva, R. y Sharma, D. (2013). Research Methods in Linguistics. Cambridge: Cambridge University Press.

Rice, K. (2006). Ethical issues in linguistic fieldwork: an overview. Journal of Academic Ethics, 4: 123155.

Rohrer, T. (2007). Embodiment and Experientialism. En Dirk Geeraerts y Hubert Cuyckens (Eds.), The Oxford Handbook of Cognitive Linguistics, 9, pp. 139-169. Oxford: Oxford University Press.

Torero, A. (2002). Idiomas de los Andes. Lingüística e historia. Lima: IFEA y Editorial Horizonte.

Turner, Mark. (2007). Conceptual Integration. En Dirk Geeraerts y Hubert Cuyckens (Eds.), The Oxford Handbook of Cognitive Linguistics, 15, pp. 377393. Oxford: Oxford University Press. 